\title{
Pathogenesis of PTSD and Its Indigenous Treatments
}

\author{
Shufan Yang, ${ }^{1, *}$ \\ ${ }^{1}$ Tianjin No. 1 High School, Tianjin 300051, China \\ *Corresponding author.Email: angela@cas-harbour.org
}

\begin{abstract}
PTSD (post-traumatic stress disorder) is a kind of disease which begins to affect modern people's lives. Previous researchers mainly focused on how to effectively recognize the symptoms of this disease, while this paper seeks to analyze the different ways to cure PTSD with three parts, including some local methods. It is necessary to seek for direct methods to cure such a kind of mental disease and some methods are actually useful. Through the author's analysis, it could be concluded that traditional Chinese medicine has a prospect for treating PTSD.
\end{abstract}

Keywords: Mental health, Threatening death, Psychology treatment, Local method treatment

\section{INTRODUCTION}

In the modern society, many people have faced various situations which may disturb their lives. Some problems are so serious that they may be influenced by them for their rest of lives. PTSD is the name of such problems. PTSD is a disorder that a person has difficulty in recovering after experiencing or witnessing a terrifying event. The condition may last months or years, with triggers that can bring back memories of the trauma, accompanied by intense emotional and physical reactions. Symptoms may include nightmares or unwanted memories of the trauma, avoidance of situations that bring back memories of the trauma, heightened reactions, anxiety, or depressed mood. Treatment includes different types of trauma-focused psychotherapies as well as medications to manage symptoms. $3 \%-4.2 \%$ of people suffer from PTSD in their mind. In fact, if PTSD cannot be effectively cured, the patients may undergo it for really a long period. Therefore, this paper will show some ways to deal with PTSD.

\section{DIAGNOSIS OF PTSD}

Specific diagnostic criteria for PTSD are as follows. There are mainly five standards to diagnose PTSD.

\subsection{Exposure to Traumatic Events}

The first one is exposure to an actual or threatened death, severe trauma or sexual violence in one (or more) of the following ways, including directly experiencing a traumatic event, witnessing a traumatic event that happens to someone else, learning of a traumatic event in a close family member or close friend. In cases of actual or threatened death, the traumatic event must have been violent or accidental. Disgusting details of repeated experiences or extreme exposure to traumatic events (e.g. human remains collected by emergency responders) also belong to this type. The police were repeatedly exposed to details of child abuse. Diagnostic standard does not apply to contact via electronic media, television, film or pictures unless such contact is work-related.

\subsection{Bad Memories}

Following the occurrence of a traumatic event, there is one (or more) of the following invasive symptoms associated with the traumatic event: recurrent, involuntary, and invasive painful memories of a traumatic event. Children over the age of six may express themselves by repeatedly playing with a theme or aspect of a traumatic event. Dreams of recurring content or emotional pain are associated with a traumatic event. Children may have scary but unrecognizable dreams. Dissociative reactions, for example, flashbacks, in which an individual feels or acts as if the traumatic event is repeated. This reaction may occur continuously, with the most extreme manifestation being a complete loss of consciousness to the present environment. Children may reenact certain traumas during the time they are playing. Intense or lasting psychological pain caused by exposure to internal or external clues to a symbolic or similar traumatic event. 


\subsection{Avoidance of Stimulus}

After a traumatic event, a person begins to continuously avoid the stimulus associated with the traumatic event, having one or two of the following conditions: avoid or try to avoid painful memories, thoughts, or feelings about a traumatic event or its high degree of relevance; avoid or try to avoid external cues (people, places, conversations, activities, objects, situations) that evoke painful memories, thoughts, or feelings about a traumatic event or its high degree of relevance.

\subsection{Negative Changes in Cognition and Mood}

Negative changes in cognition and mood associated with a traumatic event that begin or worsen after the traumatic event with two (or more) of the following symptoms: inability to remember an important aspect of a traumatic event (usually due to dissociative amnesia rather than other factors such as brain damage, alcohol and drugs); negative beliefs and expectations about yourself, others, or the world that continue to amplify (such as "I'm bad," "No one can trust," "the world is absolutely dangerous," "my entire nervous system is permanently destroyed"). A persistent cognitive distortion of the cause or outcome of a traumatic event causes the individual to blame himself or others. Persistent negative emotional states, including fear, anger, guilt, shame could significantly reduce interest in or participation in important activities. A feeling of alienation or alienation from another person and a persistent inability to experience positive emotions (for example, feelings of happiness, contentment, or love) are also some of these negative changes.

\subsection{Change in Alertness or Reactivity}

There is a significant change in alertness or reactivity associated with a traumatic event, which begins or worsens after the traumatic event, with two (or more) of the following conditions: irritability and outbursts of anger (with little or no provocation) typically to manifest themselves as verbal or physical attacks on people or objects.

\section{INDIGENOUS TREATMENTS}

\subsection{Exposure Therapy}

There is indeed a psychological treatment for PTSD that allows the client to revisit, rethink, and recognize the meaning of the trauma, which is called exposure therapy [1]. According to the principle of conditioning, when people choose to avoid the cues and stimuli that trigger fear, they become less anxious and fearful, so such negative reinforcement leads to more avoidance behavior. However, these actions, while providing short-term relief from fear, also increase the inverse association between the stimulus and the fear response, making the fear symptoms worse. According to information processing theory, if a person is repeatedly exposed to an environment that can trigger traumatic memories, but is completely safe, that person will achieve habituation and eventually change the fear schema to disassociate the situation with fear. This repeatedly exposes people to situations that trigger traumatic memories and ensure safety in the treatment of PTSD, which is the exposure therapy for PTSD [2].

Exposure therapy often takes several times, such as once a week for 9-12 weeks, 90-120 minutes each time. 1-2 times of fore shaping is also required before the formal treatment phase begins. In addition, exposure can be divided into imaginary exposure and field exposure guidance, where the duration of imaginary exposure is generally longer. In this process, the client does not need to actually go back to the place where the trauma occurred, but needs to repeatedly and vividly recall the traumatic event, and at the same time describe the situation and experience of the event aloud. In order to achieve the repeated and vivid purpose, the therapist will also ask the details: How do you feel now? What was going through your mind when you saw this picture? Describe what you smell? Does that sound painful? It is true that the visitor will feel uncomfortable when recalling and describing, but this discomfort is not dangerous, because people and things in the memory will not really appear again, and will not really hurt himself or herself again.

\subsection{Psychological Intervention}

The symptoms of PTSD and CPTSD are different and there are several differences between them. Thus the interventions are also different. Individuals of different ages experience post-traumatic stress. The coping patterns of disorders are different, so protocols should be developed for patients of different ages, which indicates targeted treatment options. Figure 1 shows the percentage of numbers in a previous study, which indicates the discrepancy between different interventions. 


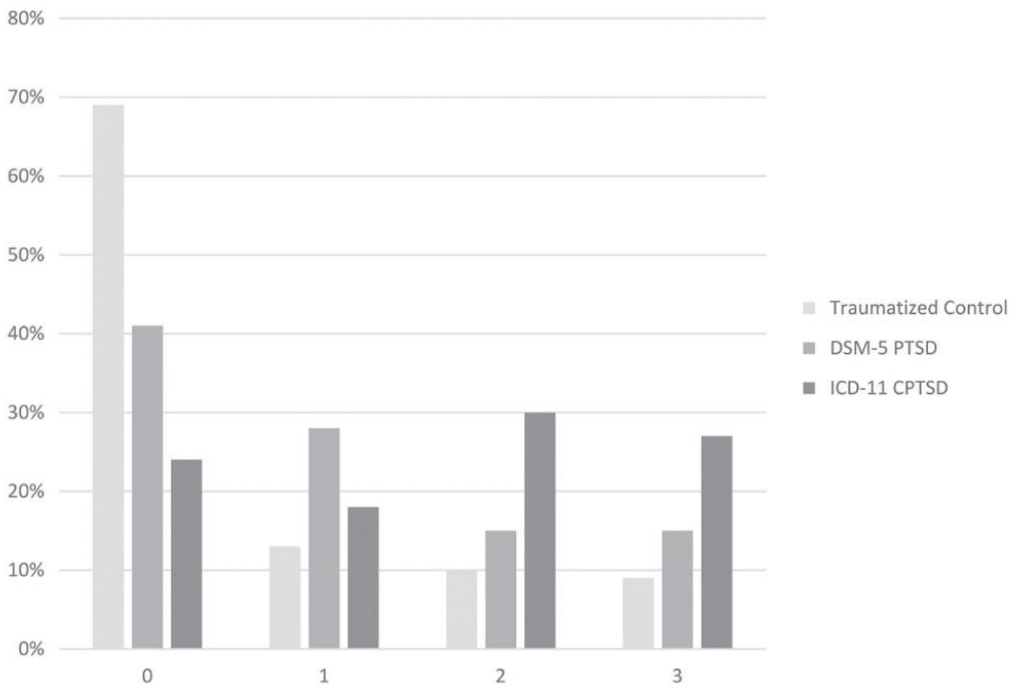

Figure 1 Percentage of number in different types of exposure to moderate-to-severe childhood emotional, physical, and/or sexual abuse by group type [3]

Currently, treatments of PTSD in adults are primarily used, such as behavioral therapy and cognitive behavioral therapy, such as acceptance therapy, prolonged exposure therapy. Methods, cognitive processing therapy and eye movement desensitization reprocessing therapy are also applied to the clinical practice. The approach for treating children differs from that of adults, often with the use of play and group therapy.

In addition to the need for PTSD interventions to help individuals with symptoms, there is also a need for other PTSD curing methods. Patients need to be assisted to rebuild their sense of autonomy and control, as well as learn skills such as emotional regulation. What is more, establishing a healthy and positive interpersonal model is also important. CPTSD serves as an independent diagnosis. The diagnosis has been proposed for a short time, and more studies are needed to select subjects with more strict and formal diagnostic criteria, so as to truly find effective methods for CPTSD intervention. At present, the therapeutic methods for adult CPTSD mainly include phased intervention mode (including stabilization stage, trauma treatment stage, consolidation and promotion stage), while the therapeutic methods for children CPTSD mainly include emotional and interpersonal skills training, ideal parent image method, narrative therapy, etc.

\subsection{Localized Treatment Methods}

Traditional Chinese Medicine has an extensive and profound culture, and there have been lots of researches and verification in China. The effectiveness of using
TCM therapy to treat patients in the field of trauma shows its unique value. Through acupuncture and moxibustion spectrum of mental and behavioral disorders, it can be found that acupuncture and moxibustion can treat sleep disorders, obstacle, anxiety disorder and obsessivecompulsive disorder. Acupuncture and moxibustion can also help to treat depression, schizophrenia and has curative effect on other diseases.

Cut belongs to acupuncture and moxibustion clinical dominant disease species. A number of clinical trials have shown that needle and moxibustion is effective in treating PTSD [3]. In addition, the modified TCM emotional therapy is based on the point that language, behavior, or deliberately arranged scenes can affect the patient's emotional and psychological well-being.

Activities could promote and improve patients' social functioning activities in order to achieve treatment and well-being. There are six ways, including solving doubts, winning emotions, shifting essence and changing qi, inducing suggestion and using rope [4]. This method is more specific and feasible compared with the psychotherapy. Under different circumstances, choosing suitable methods can better achieve the purpose of conditioning, prevention and treatment of PTSD [5].

What is more, pre-tcm affective therapy has been increasingly used in the clinical treatment of PTSD, but there is still lack of understanding of its mechanism of action [6], effectiveness and effectiveness of CPTSD intervention. Therefore, a large number of empirical studies should be conducted in the future [7]. 


\section{DISCUSSION}

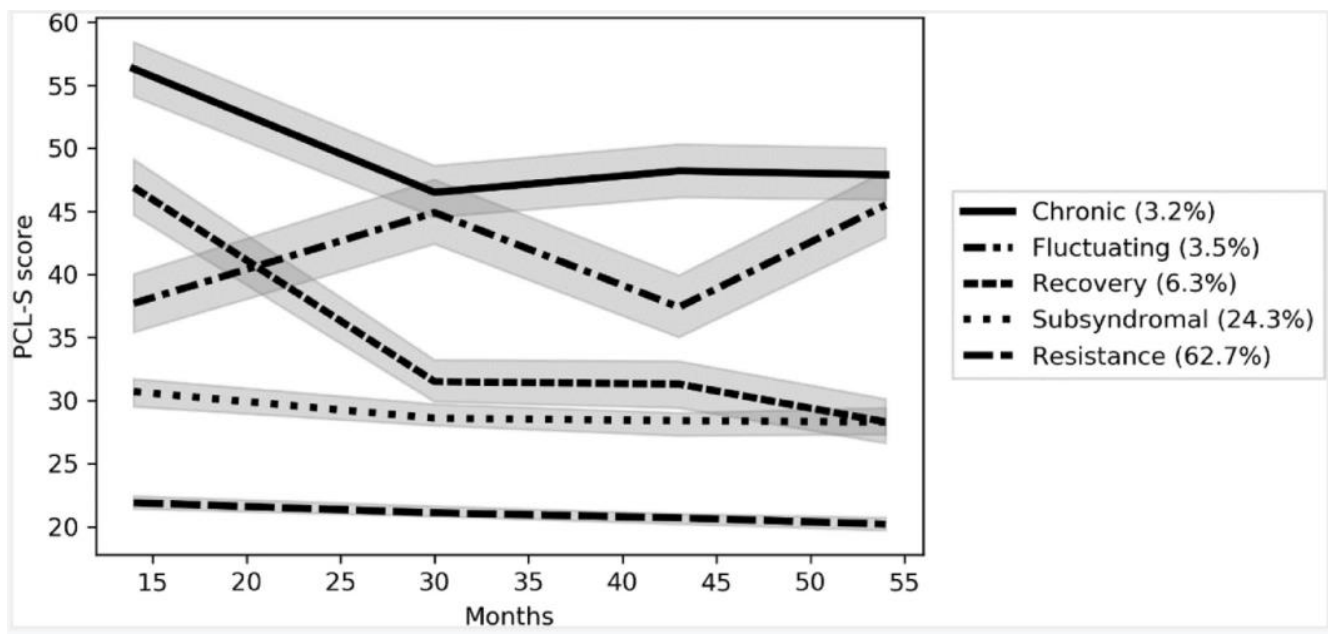

Figure 2 Trajectories of post-traumatic stress disorder (PTSD) symptoms among local disaster recovery workers following the Great East Japan Earthquake $(\mathrm{n}=745)[8]$

It can be seen in figure 2 that although the event had taken place for nearly four to five years, the effect on the people could also last for a very long time. Therefore, it is necessary to investigate more methods to treat PTSD. In addition, there are many factors that could influence the PTSD patients, and it has been proved that some of the TCM measurements could have positive effect on the treatments, such as sleep disorder of PTSD. From the author's own perspective, all of the treatment methods own their unique advantages. However, the author may suggest the local methods which apply traditional Chinese medicine is preferable because they are more effective on local patients who have PTSD.

\section{CONCLUSION}

Using treatment of traditional Chinese medicine can be effective, because it has little toxic effects with low prices, and it could improve the life quality and obviously alleviate of clinical symptoms. Traditional Chinese Medicine is also widely used in clinical practice for external treatment. However, the current studies on DR by TCM external therapy mostly focus on small sample size and short observation time without follow-up stage. There is still a lack of studies on long-term efficacy as most of the studies focus on short-term efficacy. At the same time, although traditional Chinese medicine external treatment has been used, such as injection and eye herbal fumigation, there is of no unified definition or a system with curative effect judgment standard. Therefore, we should now expand DR TCM external treatment bed in the study sample, optimize the design of experiment and improve science and treatment plan, in order to give full play to the advantages of the treatment as well as speed up the clinical application.

\section{ACKNOWLEDGMENTS}

First and foremost, I would like to show my deepest gratitude to my teachers and professors in my university, who have provided me with valuable guidance in every stage of the writing of this thesis. Further, I would like to thank my friends and parents for their encouragement and support. Without all their enlightening instruction and impressive kindness, I could not have completed my thesis.

\section{REFERENCES}

[1] J. Herman, CPTSD is a distinct entity: Comment on Resick [J]. Journal of Traumatic Stress, 2012, 25(3):256-257.

[2] American Psychiatric Association. Diagnostic and Statistical Manual of Mental Disorders DSM-IV [M]. American Psychiatric Association, 1994.

[3] A. Powers, N. Fani, S. Carter, et al. Differential predictors of DSM-5 PTSD and ICD-11 complex PTSD among African American women [J]. European Journal of Psychotraumatology, 2017, 8(1):1338914.

[4] M. Wang, G. R. Jiang, The dual representation theory and examination of the post-traumatic stress disorder [J]. Journal of psychological science, 2016, 24 (5) : 753-764.

[5] J. L. Herman, Trauma and Recovery [M]. New York: Basic Books, 1997.

[6] J. R. Freedy, H. S. Resniek, D. G. Kilpatriek, Conceptual frame work for evaluating disaster Impact: Implications for clinical intervention [M]. In Austin L S (eds.) Responding to disaster: A guide 
for mental health professionals. Washington, DC: American Psychiatric Press, 1992:6-14.

[7] T. Karatzias, M. Shevlin, P. Hyland, et al. The role of negative cognitions, emotion regulation strategies, and attachment style in complex post-traumatic stress disorder: Implications for new and existing therapies [J]. British Journal of Clinical Psychology, 2018, 57(2):177-185.

[8] A. Sakuma, et al. Trajectories for Post-traumatic Stress Disorder Symptoms Among Local Disaster Recovery Workers Following the Great East Japan Earthquake: Group-based Trajectory Modeling, Journal of Affective Disorders [J]. Vol 274, 1 September 2020, pp. 742-751. 